\title{
Methods of discovery and techniques to study endophytic fungi producing fuel- related hydrocarbons
}

\section{Author: Gary A. Strobel}

This is a postprint of an article that originally appeared in Natural Products Reports on February 1, 2014.

Strobel, G.A. (2014) Methods of discovery and techniques to study endophytic fungi producing fuel -related hydrocarbons. Natural Product Reports 39: 259-272.

http://dx.doi.org/10.1039/c3np70129h

Made available through Montana State University's $\underline{\text { ScholarWorks }}$ 


\section{Natural Product Reports}

\section{REVIEW}

1

\section{Methods of discovery and techniques to study endophytic fungi producing fuel-related hydrocarbons}

Gary A. Strobel ${ }^{*}$

Some endophytic fungi are capable of making a plethora of hydrocarbons with fuel potential. One of the major fuel-like products, among many others, is cineole as produced by Nodulisporium spp. Also, methods for measuring hydrocarbon production by microorganisms are summarized in this review as well as other information on the products,

\section{4} genomes, and potential importance of these organisms as
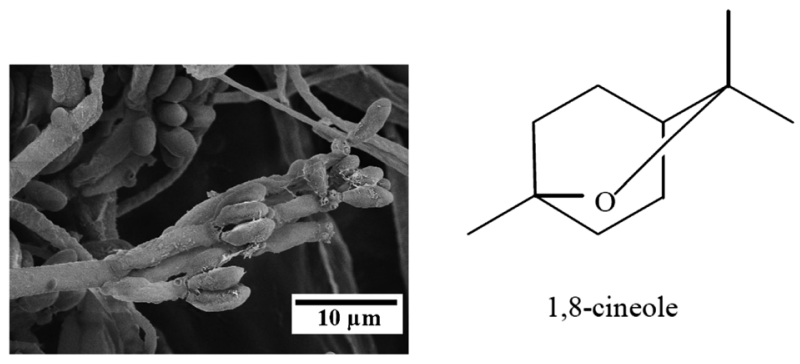

1,8-cineole

Please check this proof carefully. Our staff will not read it in detail after you have returned it.

Translation errors between word-processor files and typesetting systems can occur so the whole proof needs to be read. Please pay particular attention to: tabulated material; equations; numerical data; figures and graphics; and references. If you have not already indicated the corresponding author(s) please mark their name(s) with an asterisk. Please e-mail a list of corrections or the PDF with electronic notes attached - do not change the text within the PDF file or send a revised manuscript. Corrections at this stage should be minor and not involve extensive changes. All corrections must be sent at the same time.

Please bear in mind that minor layout improvements, e.g. in line breaking, table widths and graphic placement, are routinely applied to the final version.

We will publish articles on the web as soon as possible after receiving your corrections; no late corrections will be made.

Please return your final corrections, where possible within $\mathbf{4 8}$ hours of receipt by e-mail to: npr@rsc.org 


\section{Journal: NPR}

5 Paper: c3np70129h

Title: Methods of discovery and techniques to study endophytic fungi producing fuel-related hydrocarbons Editor's queries are marked like this... $\mathbf{1}$, and for your convenience line numbers are inserted like this... 5

Please ensure that all queries are answered when returning your proof corrections so that publication of your article is not delayed.

\begin{tabular}{|c|c|c|}
\hline $\begin{array}{l}\text { Query } \\
\text { Reference }\end{array}$ & Query & Remarks \\
\hline 1 & $\begin{array}{l}\text { [INFO-1] Please carefully check the spelling of all author names. } \\
\text { This is important for the correct indexing and future citation of } \\
\text { your article. No late corrections can be made. FOR YOUR } \\
\text { INFORMATION: You can cite this paper before the page numbers } \\
\text { are assigned with: (authors), Nat. Prod. Rep., DOI: 10.1039/ } \\
\text { c3np70129h. }\end{array}$ & \\
\hline 2 & $\begin{array}{l}\text { Is the contents entry acceptable? The descriptive text should be } \\
\text { no longer than } 30 \text { words. If the entry does not fit between the two } \\
\text { horizontal lines, then please trim the text and/or the title. }\end{array}$ & \\
\hline 3 & $\begin{array}{l}\text { A definition for "PTR-MS" has been added to the text - please } \\
\text { check that it is correct. }\end{array}$ & \\
\hline 5 & $\begin{array}{l}\text { Please check that changes to the English in this section have not } \\
\text { affected the meaning. }\end{array}$ & \\
\hline 6 & $\begin{array}{l}\text { Table } 1 \text { and 2: Please check that the data has been displayed } \\
\text { correctly. }\end{array}$ & \\
\hline 7 & $\begin{array}{l}\text { Sentence beginning "When grown on PDA...": Please check that } \\
\text { the compound names are displayed correctly. }\end{array}$ & \\
\hline 8 & $\begin{array}{l}\text { Sentence beginning "Other than sabinene...": Please check that } \\
\text { the compound names have been displayed correctly. }\end{array}$ & \\
\hline 9 & $\begin{array}{l}\text { Fig. 2: Please ensure that you have obtained the necessary } \\
\text { permissions to reproduce the image and provide any required } \\
\text { copyright text from the publisher. }\end{array}$ & \\
\hline 12 & $\begin{array}{l}\text { Ref. } 17 \text { and 38: Can this reference be updated yet with page } \\
\text { numbers or an article ID? }\end{array}$ & \\
\hline 13 & $\begin{array}{l}\text { Ref. 44: Can this reference be updated yet? Please supply details } \\
\text { to allow readers to access the reference (for references where } \\
\text { page numbers are not yet known, please supply the DOI). }\end{array}$ & \\
\hline 14 & $\begin{array}{l}\text { Please check that the GA text fits within the allocated space } \\
\text { indicated on the front page of the proof. If the entry does not fit } \\
\text { between the two horizontal lines, then please trim the text and/or } \\
\text { the title. }\end{array}$ & \\
\hline
\end{tabular}




\section{REVIEW}

Cite this: DOI: 10.1039/c3np70129h

10

Received 28th August 2013

DOI: $10.1039 / c 3 n p 70129 h$

www.rsc.org/npr
Methods of discovery and techniques to study endophytic fungi producing fuel-related hydrocarbons

Gary A. Strobel*

Covering: $2008-2013$

One promising area in the search for renewable bio-fuels is the discovery of microorganisms that produce fuel-related hydrocarbons (mycodiesel) that is in stark contrast to yeast fermentation that utilizes expensive sugars or starch to produce ethanol, which is a proven and useful source of fuel, but by no means is it ideal. Recently, a number of endophytic fungi have been isolated and described that make compounds such as mono- terpenoids, alkanes, cyclohexanes, cyclopentanes, and alkyl alcohols/ketones, benzenes and polyaromatic hydrocarbons. Many of these compounds are either identical to or are closely related to those specific classes of molecules that are found in diesel. Most importantly, these organisms make hydrocarbons while utilizing cellulosic polymers found in all plant-based agricultural wastes. Also discussed are some novel methods and techniques to quantitatively and qualitatively study hydrocarbon production by these microbes. Two models are discussed for identifying potential fuel-related compounds, scaling up production of them and advanced engine testing. Finally, it seems possible that endophytic fungi may have an additional attribute of having contributed to the formation of crude oil in the first place and a description of the paleobiosphere, to test this hypothesis, is in this review.

\section{References}

\section{Introduction}

As the world's liquid fossil fuel sources are ever diminishing, the search for alternative sources of energy is becoming increasingly important. One of the most effective ways to search for unusually metabolically active microorganisms, producing a wide diversity of secondary metabolites, is to look for these organisms in unique ecological niches. Certainly, one niche that is relatively unexplored is the living tissues of higher plants. The microbes living in these niches are known as endophytes and most commonly the greatest abundance of microbes isolated from these niches are fungi. It has recently been discovered that some of these fungi produce fuel-related substances. These new sources of fuel-like compounds are renewable and compatible with existing engine infrastructure, which is based on drop-in fuel technology. In addition, these microorganisms also possess the capabilities of growing and producing gaseous products on agricultural and other cellulosic wastes rather than valuable agricultural products that may also serve as food and feed sources. It has been discovered that certain endophytic fungi produce bioactive volatile products and many of these compounds are fuel-related. Some reviews on the bioactivity of 
endophytic microorganisms producing such volatile products have already appeared. ${ }^{1-3}$ However, uniquely, the main theme of this review is the discovery of endophytic fungi producing fuelrelated products and methods that have been developed to study and domesticate them.

Endophytic fungi are found throughout nature as they inhabit the interstitial spaces of plant tissues and cause no apparent outward evidence of their presence in the plant. ${ }^{4}$ Endophytes, at this point, represent a small fraction of the nearly 500000 known fungal species. They are associated with the roots, stems, leaves, flowers and fruits of plants and usually, upon sampling, one or more of these tissues will yield an endophyte. The enormous vastness of the plant kingdom ( $c a$. 300000 species) is only outweighed by the sheer diversity and numbers of the microbial floral species existing in these niches, which is a great deal more. Ecosystems exhibiting the greatest plant diversity also seemingly exhibit the greatest abundance and diversity of endophytic populations. ${ }^{5}$ Rainforests have been targeted for numerous searches for endophytic microbes as they claim over $60 \%$ of the world's terrestrial biodiversity, yet endophytes also exist in oceanic species such as kelps and the roots of mangroves and other marine associated plant species. Ultimately, biological diversity implies chemical diversity as constant chemical innovation is required in such highly competitive environments. It is also worthy to note that finding taxonomic novelty among organisms living as endophytic species is extremely likely. ${ }^{6}$

Apparently, the relationship existing between the microbe and the plant can vary from symbiotic to borderline pathogenic. While the endophyte needs nutritive sources offered by the host plant, its contribution to the interaction may be one of tissue protection generated by virtue of its own anti-pest metabolites. In fact, numerous bioactive natural products, including antibiotics, antimycotics, insecticides, anticancer agents and antiviral compounds have been isolated from various endophytic microbes. ${ }^{5,7,8}$ These compounds vary widely in both their

40

45

50

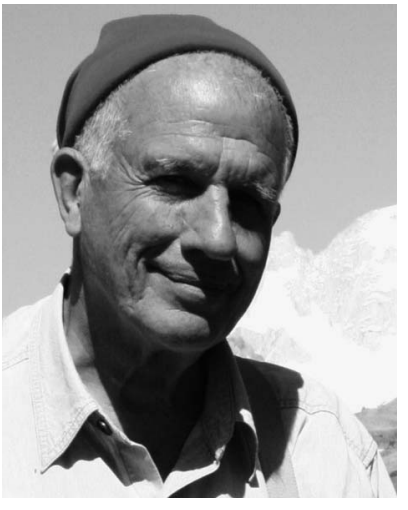
corresponding host plants. His studies have taken him to virtually all of the world's jungles and temperate rainforests. He has received an NIH career development award, the E. O. Wilson award, the Wiley award, and the R. G. Gray endowed chair for research excellence. He is a fellow of the American Academy of Microbiology and elected fellow of the World Explorer's club. chemical class as well as their complexity having such representative substances as peptides, substituted peptides, phenolics, glycosides, terpenoids, complex lipids and completely odd and unanticipated structures as tetramic acids and (3carbamoyl-quinoxalinium) chloride. It is also apparent that the endophyte and, perhaps the plant, each utilize products of the other partner to genetically regulate aspects of growth, metabolism and even the sexual cycle. The molecular biological aspects of these interactions are becoming better known.

The formulation of the concept that endophytic fungi can produce fuel-related hydrocarbons is directly traced to the discovery of a novel endophytic fungus that has been described and named as Muscodor albus. ${ }^{9}$ To date, all members of this genus produce volatile biologically active molecules that inhibit and or kill pathogenic fungi and bacteria, and some isolates of this fungus are also detrimental to insects., ${ }^{2,3}$ Because of the broad-range and effective nature of its volatile organic compounds (VOCs), this fungal genus has been comprehensively studied and it is being developed for use as an agent in biological control. ${ }^{2,3}$ In fact, artificial mixtures of the fungal VOCs, made via bacterial fermentation technologies, are now on the market. ${ }^{3}$ Thus, with the advent of the discovery of this unique fungus, it became obvious that its possible role in the contribution to the host plant-microbe interaction is to provide protection to its host via the production and release of bioactive volatiles. Then, the search for other Muscodor began via the use of the original culture of $M$. albus in a plate selection technique. ${ }^{10}$ Interestingly, $M$. vitigenus, $M$ crispans, $M$ sutura, and $M$. roseus all appeared as new species of this fungus and others began to be reported from other locations around the earth. ${ }^{3}$ Just as important, however, was the appearance of other gasproducing fungi, on plates with $M$. albus, having total resistance to the M. albus volatiles while at the same time making their own unique sets of gases, most of which also possessed antibiotic activities. These organisms turned out not to be species of Muscodor, but other fungal endophytes that normally inhabit wood. In addition, examination of the chemistry of the volatile substances produced by these endophytes, revealed the presence of alkanes, alkenes, alkyl esters, benzene derivatives, terpenoids, polyaromatic hydrocarbons and other compounds that are either identical to or are related to the chemical component families of diesel fuel. For this reason, the volatiles were given the name "mycodiesel" and the concept that endophytic fungi can produce fuel-related substances was proposed. ${ }^{11}$

Thus, this review deals with the discovery of a number of endophytic fungi that make VOCs with both bioactivity and potential as fuels. This is a paradigm that dramatically differs from other approaches that utilize microbial secondary products, such as ethanol, and other small molecular weight alcohols as fuels. ${ }^{12}$ Furthermore, the bioengineering of common microbes to produce these alcohols or fatty acids with fuel potential is also not the focus of this approach. Instead, this review highlights the distinct possibility that there are a plethora of microbes, in nature, that uniquely possess the metabolic machinery to produce representative compounds (VOCs) commonly associated with each of the groups of 
hydrocarbons that make up diesel fuel and other fuels such as gasoline and jet $\mathrm{A}$. These organisms also intrinsically have the capability to utilize cellulose, hemicellulose and other relatively inert natural polymers to produce these hydrocarbons. Also, these organisms have the potential to be bioengineered to enhance their production of individual fuel components. Some techniques and methods used to find these organisms, analyze their products, and study their biology are also included in this review, along with specific examples of microbes that carry out hydrocarbon production. It should be noted that all chemical nomenclature in this report follows that of the NIST database, from which all VOC data have been retrieved.

\section{The discovery of hydrocarbon- producing fungi}

At the outset it was realized, because of their unique position and role in nature, that endophytic microbes should be selected and examined for their ability to make hydrocarbons with fuel potential. Endophytes, while mostly having been studied from terrestrial sources also usually have distinct counterpart species that inhabit both fresh and salt water plant species. Unquestionably, they are the first microbes to begin the processes of plant decay. ${ }^{11,13}$ However, when they are in the endophytic state, they only occupy a minimal area of the interstices of the host plant. It is not clear what agent or agents keep the growth of the endophyte suppressed whilst in this restricted stage of development, but tannins and tannin-like substances are strongly suspected. ${ }^{14}$ Thus, upon the demise of the host plant, either by leaching or degradation, those factors regulating the growth of the endophyte are removed and the microbes begin to grow within the tissue that it originally inhabited. ${ }^{14}$ Virtually all plants have one or more endophytes associated with them. ${ }^{5}$ Certainly, not all of these microbes will be making the products of interest, in fact, in a preliminary screen of endophytic fungi of oil-palm making volatile organic substances (VOCs), only about one half made any detectable vOCs at all [Green, SGI, unpublished data]. Usually, it is also the case that fungi making a plethora of VOCs also have antimicrobial activity associated with these gases. Thus, as searches have been conducted for endophytes making antimicrobial VOCs it has become obvious that some of these compounds were either identical or related to substances normally found in diesel fuel.

\subsection{Isolating and screening endophytes making hydrocarbons}

To date, at least 500000 fungal species have been described. Focusing on just endophytic species for hydrocarbon production greatly restricts the potential spectrum of fungal genera likely to be isolated. Nevertheless, as initially stated in this review, a case has been made to select and examine the world of endophytes for this purpose. The endophytes can be obtained using standard techniques of collection, storage and isolation to pure cultures. It begins by placing ethanol treated plant tissues on a hard surface and carefully removing internal tissues, which are then placed on Petri plates containing water agar. Eventually the endophytes emerge from this tissue and are picked and transferred to potato dextrose agar (PDA) Petri plates. ${ }^{5}$ It is to be noted that the greatest diversity of microbes will most likely be obtained from tropical or temperate rainforests. ${ }^{5}$ However, once in hand there are several sophisticated and not so sophisticated procedures that can be used to target those microbes that are making hydrocarbons as outlined below.

\subsection{SPME fiber screening}

Specific endophytes may be grown in small vials on various agar media for various lengths of time and then robotically sampled by a system directly attached to a GC-MS comparable to those made by Agilent. The SPME (solid phase micro extraction) fiber method uses a solid state collection matrix on a small fiber to sample the volatiles in the gas phase over the microbial culture. ${ }^{11}$ In this manner each organism receives comparable attention relative to its ability to make the products of interest. One difficulty is that the SPME technique has some limitations when used with complex samples containing VOCs with very different physical properties due to the differential adsorption efficiencies of the VOCs on the SPME fiber and thus cannot be accurately used in a quantitative manner. In addition, since the sample volume is small in these automated systems, it represents a non-concentrated mixture of products. Thus, some compounds that are made by the fungus in small concentrations may be missed in the analysis. Finally, the technical problems in handling a large number of samples in this manner, setting up the system and dealing with large data sets may preclude its practical use.

On the other hand, a simple technique can be used on individual culture plates by drilling a hole in the side of the plate to insert the SPME fiber. Regardless of what plate geometry is used, it is exceedingly desirable to use authentic standards to help confirm the identity of any fungal compound as well as heavily relying on the percentage match of the unknown to the NIST or other database authentic standard compound. ${ }^{5,11}$

\subsection{Muscodor albus selection technique}

The discovery of the fungus first proposed as a producer of fuelrelated hydrocarbons was accomplished via the use of the endophytic fungus M. albus. The sole appearance of Gliocladium roseum (now classified as Ascocoryne sarcoides) in Petri plates (PDA) supporting the growth of $M$. albus shows that this fungus was able to survive and grow in the presence of the inhibitory and lethal VOCs of M. albus. ${ }^{15}$ Further testing of the recovered $A$. sarcoides culture revealed that its VOCs were active against other test fungi and that some of its volatile products had fuel potential. ${ }^{11}$ Since those observations were made it has become increasingly evident that many other endophytic fungi which produce volatile hydrocarbons are also resistant to $M$. albus VOCs and their VOCs are biologically active. ${ }^{16}$ Thus, this selection technique has the potential of eliminating $80-90 \%$ of endophytic microbes that probably would not be of interest relative to VOC production, since they would succumb to the VOCs of $M$. albus while growing out of the plant tissues on the 
Petri plates. This technique has merit if one does not wish to deal with a plethora of microbes that may not be of interest. Nevertheless, some important hydrocarbon-producing fungi are sensitive to the VOCs of M. albus and would have been missed if this technique had been exclusively used as a microbe selection technique. ${ }^{17}$

\subsection{Human olfactory detection methods}

Usually, after 10-14 or more days of incubation on PDA, most VOC-producing fungi will begin to exude gaseous products. Many of these VOCs have a distinctive odor that can be readily perceived by the human olfactory system. ${ }^{16}$ Thus, simple smell tests can and should be conducted by someone with a sensitive nose on each fungal culture in the emerging collection of endophytes. ${ }^{16}$ For instance, many of the terpenoids can easily be detected by their distinctive odor. As an example, 1,8-cineole, which is the subject of a number of papers on hydrocarbonproducing fungi, has the smell of a eucalypt forest and this product is commonly termed eucalyptol. ${ }^{16}$ Mixtures of other terpenoids, esters, ketones, and some alkanes also will be odoriferous and such cultures should eventually be targeted for SPME analysis. This simple "smell" test can be the first clue in selecting a culture for its ability to make VOCs, which can then be followed with more specific chemo-analytical testing. Obviously, the chief limitation of the technique is that it is only provides an initial selection tool in picking organisms for further study and some individuals may have rather poor olfactory detection capabilities making fungal plate selection impossible.

\section{Analytical methods for fungal hydrocarbons}

Quite unlike the large number of techniques and instrumentation that are available for standard natural products chemistry, there is a relative dearth of tools available to do accurate measurements on VOCs that are hydrocarbon based from microbial fermentation. Thus, since the production of hydrocarbons by fungi is a relatively new observation, the methods for analyzing these compounds have been borrowed from other applications. One of the most frequently used methods, in fungal studies, for obtaining a qualitative assessment of the VOCs being produced by the fungi is the SPME method mentioned above. However, quantification of the VOCs is extremely important, especially for reasons relating to the potential commercialization of the products. Some analytical methods for determining hydrocarbons are described below:

\subsection{Stainless steel column Carbotrap technology}

A novel technology has been devised that utilizes the Carbotrap materials B\&C. A uniquely designed stainless steel column was built and the Carbotrap materials B\&C were placed into each of two compartments, respectfully (Bozeman Scientific). ${ }^{18}$ These materials were selected because of their trapping specificity for hydrocarbons and hydrocarbon derivatives. These well described materials produced by Supelco are commonly used in therma-desorption tubes with direct input into GC-MS systems. In this case, they have been adopted in a special manner as semi-preparative column materials. ${ }^{18}$ Recently, it has been learned that a properly sized carbon-black material can also be used to trap hydrocarbons in the stainless steel column. Because this material is so inexpensive, the costs to carry out hydrocarbon trapping experiments using this technology can be greatly reduced [Strobel, Blatt, Booth, unpublished results]. A bioreactor has also been constructed which allows sterile air to be over and around the fungal culture and then when it exits the flask it passes through the Carbotrap column. The column, when properly dehydrated by several gentle heating cycles in the presence of dry nitrogen gas being passed through it, is weighed and the total hydrocarbons trapped is determined. The column technique is capable of providing $95-99 \%$ trapping efficiencies on various terpenoids and hydrocarbons that have been tested. Recovery of the materials from the column by heating a programmable oven with trapping in a vial bathed in liquid nitrogen will give recoveries in the range of $65-70 \% .^{18}$ The individual products in the mixture can then be determined by the SPME fiber technique described above.

\subsection{Proton transfer reaction mass spectrometry}

This mass spectral technique can be used on-line, in real time to quantify the production of individual fungal volatiles on a continuous monitoring basis. ${ }^{16,19,20}$ It is to be recognized that the proton transfer reaction mass spectrometry (PTR-MS) instrument ionizes organic molecules in the gas phase through their reaction with $\mathrm{H}_{3} \mathrm{O}^{+}$, forming mostly protonated molecules $\left(\mathrm{MH}^{+}\right.$, where $\mathrm{M}$ is the neutral organic molecule), which can then be detected by a standard quadrupole mass spectrometer. ${ }^{16,19,20}$ This process can be run on air samples with or without dilution, since the primary constituents of air (nitrogen, oxygen, argon and carbon dioxide) have a proton affinity less than water and thus are not ionized and not detected. Most organic molecules (excepting alkanes) have a proton affinity greater than water and are therefore ionized and detected. ${ }^{20}$ For instance, this would include hydrocarbons with double or triple bonds, and compounds bearing one or more oxygen atoms. A further advantage of PTR-MS is that from the known or calculated quantities, the reaction time, the amount of $\mathrm{H}_{3} \mathrm{O}^{+}$present, and the theoretical reaction rate constant for the proton transfer reaction, the absolute concentration of constituents in a sample can be quantified..$^{\mathbf{2 0} 21}$ The operator is obligated to have prior knowledge of the ion signals generated by the molecules of interest, however, and this means that some prior knowledge of the organic constituents in the head space of the culture are known and what specific ions that they will generate. Then the ion signals in the PTR spectrum can be assigned to specific compounds known to be present in the mixture. Finally, as mentioned above, an enormous advantage of PTR-MS is that it can be run in real time, and thus it will continuously produce data on the concentrations of specific ions of interest over the entire course of the fermentation period. ${ }^{16,19}$

Now, most recently, a PTR-TOF mass spectrometer has been used to identify and quantify compounds in the gas phase of 
Hypoxylon sp. [Knighton and Strobel, unpublished results]. This emerging technique holds enormous promise as a means of accurately identifying and at the same time quantifying compounds in the gas phase in microbial fermentations. Again, initially, some information on the nature of the compounds present, as obtained by SPME analysis, is desirable in order to acquire identifiers for specific ions generated by the PTR-TOF.

\subsection{Nuclear magnetic resonance spectroscopy}

A very usable and quick method of making a total measure of hydrocarbons in solution is via proton NMR spectroscopy. This method relies on the fact that proton measurements in the NMR are quantitative. This method has been used by a number of investigators in making hydrocarbon measurements of fungal hydrocarbons. In one case, after methylene chloride extraction of the gaseous foam of a Myrothecium sp. culture the total hydrocarbon concentrations were calculated. ${ }^{22}$ This was done by integrating the total proton signal in the areas of the NMR spectrum having hydrocarbons and the total signal of protons in methylene chloride. The calculation for the actual amount of hydrocarbons (HC) present is as follows:

$$
\mathrm{HC}=V\left(P_{\mathrm{CH}_{2} \mathrm{Cl}_{2}} / P_{\text {fungal }}\right)\left(\text { Area fungal } / \text { Area } \mathrm{CH}_{2} \mathrm{Cl}_{2}\right)
$$

$$
\mathrm{HC}=\text { fungal VOCs }
$$

$V=$ volume of concentrate

$P_{\mathrm{CH}_{2} \mathrm{Cl}_{2}}=$ number of protons in $\mathrm{CH}_{2} \mathrm{Cl}_{2}$

$P_{\text {fungal }}=$ number of protons in fungal volatiles obtained by averaging the protons per molecule of those compounds detected by GC-MS estimated at 8.6.

Area $=\mathrm{NMR}$ integral area for sample $\mathrm{HC}$ or $\mathrm{CH}_{2} \mathrm{Cl}_{2}$ as indicated

\section{What is diesel?}

Since this discussion relates to fungal products that are identical or related to substances found in diesel it is worthwhile to be reminded the diesel fuel is obtained by heating crude oil to $250-350{ }^{\circ} \mathrm{C}$ for fractional distillation purposes. The product contains a series of compounds ranging from about C-5 to C-21. Analyses of diesel fuels obtained in different parts of the world reveals that, while each are unique, all contain representatives of four major classes of compounds for purposes of this discussion. These are represented, for purposes of this review, by Class 1 - which are the straight chain and branched alkanes (pentane, hexane, heptane, octane, etc., and branched hydrocarbons such as 3-methyl nonane and many others). Class 2 compounds are the cyclic alkanes and their derivatives, exemplified by cyclohexane, cyclopentane and a number of cycloalkanes decorated with various hydrocarbon side chains. Class 3 compounds are represented by a large number of benzene derivatives having from 0 to all carbon atoms in the ring

possessing a hydrocarbon side chain. Finally, Class 4 is a large group of substances that qualify as poly-aromatic hydrocarbons, which are represented by well hydrogenated naphthalene and azulene-like substances. ${ }^{23}$ In addition, other miscellaneous heterocyclic substances are also found in diesel. ${ }^{23}$

\section{Some specific examples of hydrocarbon-producing endophytes}

While a number of reports have appeared in the literature concerning the production of various volatile compounds by fungi in general, it appears that until relatively recently, no one has made the connection between fungal VOC production and the potential that fungi may have for fuel production. ${ }^{11}$ Many studies on fungal volatiles have been done on microbes that invade moist walls or are products of the fungal induced decay of wood. ${ }^{24}$ Now, a much wider range of fungi have been discovered that make compounds with fuel potential.

\subsection{The case of Gliocladium roseum (Ascocoryne sarcoides)}

An endophytic fungus, Gliocladiun roseum (NRRL 50072) was isolated from Eucryphia cordifolia growing on the Pacific coast slopes of central Chile. The selection technique, using M. albus on a split culture plate, was used to quickly acquire this organism. This isolate of G. roseum itself produced volatiles with antibiotic properties. ${ }^{15}$ Some of these volatiles were represented by a series of hydrocarbons and hydrocarbon derivatives on an oatmeal-based agar under microaerophilic conditions, as analyzed by SPME-GC-MS. Most strikingly in the VOC mixture was a series of the acetic acid esters of straight chained compounds including those of hexyl, heptyl, octyl, secoctyl and decyl alcohols. ${ }^{11}$ Other independent laboratories have since confirmed this finding using an identical organism growing on the same and other substrates, and in other conditions, but with differing results relative to the production of straight chain and branched hydrocarbons..$^{25,26}$ Nevertheless, this was the first clue that an endophytic fungus had the metabolic capacity to produce compounds with the structural potential of being converted into the main ingredients of the Class 1 alkanes that are found in all diesel fuels. However, various straight chained (free form) compounds have also been reported for this fungus, including compounds such as heptane, octane, 2- pentene, dodecane. ${ }^{25,26}$ Also found in the culture head space of this fungus are a number of cycloalkanes including 1-methyl cyclohexene, cyclodecane and cyclodecene, and these cycloalkanes are representative of the Class 2 hydrocarbons as discussed above. ${ }^{25,26}$ The authors, in the original report also indicated that the G. roseum was producing a mixture of branched hydrocarbons. ${ }^{11}$ This was an important discovery given the fact that these hydrocarbons are energy dense. Further analyses of the mass spectral data indicated that many of these assignments may have been the subject of misinterpretation. ${ }^{27}$ Nevertheless, new work has added confirmation that the organism can produce branched alkanes, depending upon the substrate being used, but still with differing final conclusions on the size of the molecule and the 
extent of branching. Such compounds as hexane, 3-methyl, 1,4hexadiene, 3-ethyl, hexane, 3,3-dimethyl, nonane, 4,5-dimethyl, undecane, 3-methyl, 1- heptane, 6-methyl, among others are produced by this organism. ${ }^{25,26,28}$

The most comprehensive fermentation work on A. sarcoides, using cellulose, glucose and potato dextrose broth in comparative studies, indicated that fuel-range organics were produced by this organism on each substrate. ${ }^{26}$ However, yields of dieselrange hydrocarbons on cellulose were the highest of the various substrates tested at $45 \mathrm{mg}$ per $\mathrm{g}$ biomass, and the lowest for gasoline range hydrocarbons at $0.12 \mathrm{mg}$ per $\mathrm{g}$ biomass. $^{26}$ Overall, the largest classes of compounds produced on all three substrates were the aromatics and alkanes. Other organics including esters, alcohols, ketones, ethers, terpenes, and acids were also observed and some of these also have potential as fuels. ${ }^{11,25,26}$

Quantification of VOCs produced by NRRL 50072, grown on oatmeal agar, as measured by PTR-MS, resulted in a level of detectable organic substances in the air space of the oatmeal agar medium in the order of $80 \mathrm{ppmv}$ (parts per million by volume) in a 18-day old culture. Scaling the PTR-MS profile the acetic acid heptyl ester was quantified at $500 \mathrm{ppbv}$ and subsequently the amount of each compound in the GC-MS profile could be estimated and all yielded a total value of $c a .4 .0 \mathrm{ppmv}$. Measurements made by PTR-MS demonstrated its value in estimating the production capability of volatiles by microorganisms. ${ }^{\mathbf{1 1}}$

It is to be stressed that it is extremely important to do critical taxonomic studies on any potential hydrocarbon producer. This will eventually allow for the ultimate association of microbial relationships that are currently, or in the past, may have been responsible for hydrocarbon production. As an example, it was originally reported that the fungus producing hydrocarbon derivatives as isolate NRRL 50072 was classified as G. roseum strain C-13, primarily based upon its morphological characteristics and at a time prior to the expansion of GenBank entries of many fungal ITS rDNA-sequences. ${ }^{11,15}$ Specifically, it produced

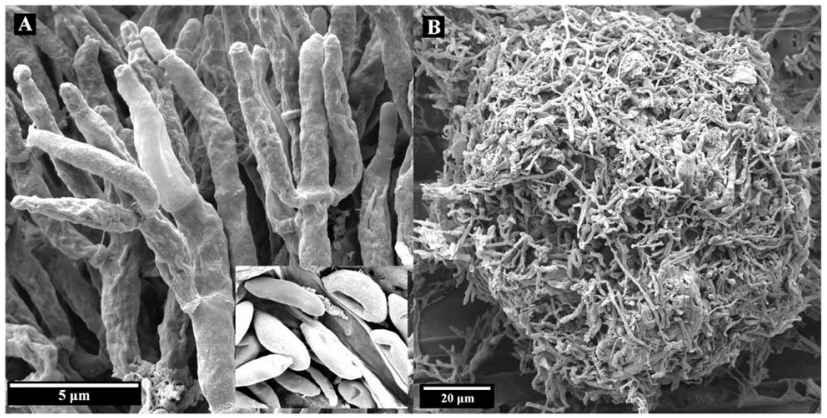

Fig. 1 Scanning electron micrographs of G. roseum strain NRRL 50072. (A) $=$ The condiophores and phailides share commonalities in shape, size, and appearance to $A$. sarcoides strain $A V-70 .{ }^{29}(B)=$ One of a few synnemata of NRRL 50072 that formed on the sterilized stems of its host, Eucryphia cordifolia and were poorly developed, irregular and lacked a well defined hymenial layer . The insert = the conidia of NRRL 50072. The ITS region of this organism has a match to that of $A$. sarcoides and thus NRRL 50072 is an imperfect stage of this fungus. ${ }^{29}$ slimy clumped conidia on verticillated conidiophores and red pigmented particles in culture (Fig.1). This feature is characteristic for G. roseum. However, DNA sequence analysis (ITSrDNA) suggested a closer match, at a 99\% level, to Ascocoryne sarcoides (Heliotiales) than to those fungi assigned to the group of Gliocladium spp. (Hypocreales) and this was originally reported but there was no morphological evidence to support the taxonomic assignment to A. sarcoides. ${ }^{11}$ However, comparative genetic and morphological studies confirmed that the anamorphic stage of an authenticated culture of $A$. sarcoides strain AV-70, obtained directly from the field, shared many of the same morphological, and genetic features as NRRL 50072, but the latter was unable to produce synnematal masses unless it was presented with a proper substrate. ${ }^{29}$ This suggests that NRRL 50072 is most likely a form of the imperfect stage of $A$. sarcoides, namely a Coryne sp. Serial transfer of the fungus AV70 resulted in major cultural changes in the fungus especially in the production of aerial hyphae, pigment production and the formation of synnemata. ${ }^{29}$

Since A. sarcoides produces potential-biofuel metabolites when grown on a cellulose-based medium, the genetic pathways needed for this production are unknown and the lack of genetic tools makes traditional reverse genetics difficult. ${ }^{28}$ Therefore, the genomic characterization of $A$. sarcoides using transcriptomic and metabolomic data has been done. ${ }^{28}$ The data were used to establish a hypothetical base for biofuel production pathways. In total, almost 80 biosynthetic clusters were identified, including several previously found only in plants. Additionally, many transcriptionally active regions outside of genes showed condition specific expression, offering more evidence for the role of long non-coding RNA in gene regulation. The analyses and datasets, now completed on A. sarcoides, contribute to the study of cellulose degradation and biofuel production and provide the genomic foundation for the study of a model endophyte system involving this fungus. The workers in this group have provided the highest quality fungal genomes and the only thoroughly annotated and transcriptionally profiled fungal endophyte genome currently available. ${ }^{28}$ The analyses and datasets contribute to the study of cellulose degradation and biofuel production and provide the genomic foundation for the study of a model endophyte system.

Nevertheless, other workers have acquired Gliocladium spp. isolates from a National Collection and proceeded to examine them for hydrocarbon production. ${ }^{13}$ It is to be noted that the authentic Gliocladium spp. they acquired is most likely to be the imperfect stage of either Nectria spp. or Hypocrea spp. and thus, not necessarily related to the NRRL $50072 .^{30}$ They also observed that the Gliocladium spp. isolates produced C6-C19 hydrocarbons directly from cellulosic biomass and they showed the presence of Class 1 and Class 3 hydrocarbons from these fungi. Compounds such as hexane, heptane, 1-octene, hexane, 3,4dimethyl, nonane, 3-methyl, along with dodecane, tridecane, hexadecane, nonadecane and benzene were found in the gas phase of the cultures. However the production of hydrocarbons was nearly doubled when the fungi were co-cultured with E. coli. No precise quantitative data on hydrocarbon production by these fungi was offered by these authors. 


\subsection{The case of Hypoxylon sp. making hydrocarbons}

An endophytic fungal strain of Hypoxylon sp. (CI-4A) was isolated from Persea indica an evergreen tree native to the Canary Islands, where it grows not in abundance but is found on several islands including Tenerife in the Laurisilva. ${ }^{16}$ This organism was isolated as its imperfect stage as Nodulisporium sp. from a small stem in the crown of the tree and it readily grows in the presence of the VOCs of M. albus, which should facilitate its isolation from other plant sources. When grown on PDA-Petri plates, the VOCs produced by this fungus were primarily 1,8-cineole, 1-methyl-1,4-cyclohexadiene, and (+)- $\alpha$ methylene- $\alpha$-fenchocamphorone. Not only these, but many of the compounds made by this organism are of interest because of their high energy densities and thus the potential they might have as mycodiesel fuels. ${ }^{16}$

Six-day old cultures of Hypoxylon sp. displayed maximal VOC-antimicrobial activity against Botrytis cinerea, Phytophthora cinnamomi, Cercospora beticola, and Sclerotinia sclerotiorum, suggesting that the VOCs may play some role in the biology of the fungus and its survival in its host plant. In fact, this discovery has implications in developing methodology for strain improvement via mutation /selection techniques. ${ }^{\mathbf{1 6}}$ Media containing starch- or sugar-related substrates best supported VOC production by the fungus. Continuous direct on-line quantification of VOCs was measured by PTR-MS, covering a 12-day range with optimum VOC production occurring at 6 days at $145 \mathrm{ppmv}$ with a rate of production of $7.65 \mathrm{ppmv} \mathrm{h}^{-1} .^{15}$ The production of 1,8-cineole from a fungal source is of significant interest given the fact that this compound does not have any previously known biological sources aside from plant tissue, and has thus far limited the compound's availability for fuel purposes.

Biosynthesis of 1,8-cineole, in plant tissues involves its conversion from geranyl pyrophosphate by 1,8-cineole cyclase (cineole synthase), whose activity is inhibited by cysteine- and histidine-directed reagents but protected by substrate-metal ion complexes, with the ether oxygen atom of this oxygencontaining terpene being solely derived from water. ${ }^{31}$ However, molecular biological studies on Hypoxylon sp. have indicated that its cineole synthase is not identical to the one in plants [Schaible and Strobel, unpublished results]. Nevertheless, an understanding of these individual pathways and their derivation from a common pathway involving production of geranyl pyrophosphate from mevalonate (MVA pathway) agrees with the idea that Hypoxylon sp. may be conditioned for biosynthesis of monoterpenes and subsequent manipulation of these pathways could lead to their optimum production on a mass commercial scale.

Previous studies on 1,8-cineole have shown the prevention of phase separation when used as an additive in ethanol-gasoline fuel blends. ${ }^{32}$ Furthermore, when fuels comprised of a gasolineeucalyptus oil mixture (with 1,8-cineole as the major fuel component up to $80 \%$ or more) there was an improved octane number and reduced carbon monoxide exhaust. ${ }^{33}$ Thus, 1,8cineole is a worthy target molecule for study and scale up and it has amazing potential for replacing fossil-based hydrocarbons as a fuel additive.

There may be factors controlling 1,8-cineole and other hydrocarbon molecule production in this fungus, based on the observation that serial transfer of the fungus on PDA resulted in a dramatic reduction of VOC production including 1,8-cineole. Then, when the attenuated organism was placed on certain plant parts and plant extracts, the VOC production resumed to normal as measured by PTR-MS [Nigg and Strobel, unpublished results]. This observation is suggestive of one of more regulatory mechanisms involved in hydrocarbon production that seems to be influenced by the host plant. In addition, experimental work was undertaken to affect the gene expression of this organism through compounds known to act as epigenetic modifiers and explore the possibility of the appearance of new products and/or the increased or decreased production of other VOCs already known from this organism. For instance, it is realized that the complete analyses of fungal genomes in recent times has indicated that many putative biosynthetic gene clusters are located in the distal regions of the chromosomes and exist in a heterochromatin state with the constitutive genes often transcriptionally controlled by epigenetic regulation such as histone deacetylation and DNA methylation. ${ }^{34}$ Several studies have demonstrated that the inhibition of histone deacetylase activity, through gene disruption or use of epigenetic modulators, leads to the transcriptional activation of gene clusters resulting in enhanced production of secondary metabolites. ${ }^{34,35}$ Fungi treated with DNA methyl transferase- and histone deacetylaseinhibitors exhibited natural product profiles with enhanced chemical diversity, demonstrating that the small-molecule epigenetic modifiers are effective tools for rationally controlling the native expression of fungal biosynthetic pathways and generating biomolecules not previously associated with the organism. ${ }^{35,36}$ Thus, the fungus was exposed to the epigenetic modulator histone deacetylase and DNA methyl transferase inhibitor SAHA (suberoyl anilide hydroxamic acid) as well as 5azacytidine (AZA). Subsequently, the organism displayed striking cultural changes including variations in pigmentation, growth rates and odor, in addition to significant differences in the bioactivities of its VOCs. ${ }^{37}$ Analyses (GC-MS) of the VOCs produced by the modulated fungus showed considerable variation with the emergence of several compounds not previously observed in this fungus, particularly an array of tentatively identified terpenes such as $\infty$-thujene, sabinene, $\delta$-4-carene, $\gamma$ terpinene, $\infty$-terpinolene and $\beta$-selinene, in addition to several primary and secondary alkanes, alkenes, organic acids and derivatives of benzene (Table 1 ). ${ }^{37}$ The spectrum of identifiable compounds, in this study, was greatly enhanced by virtue of being collected and trapped by a Carbotrap (as mentioned above) resulting in cleaner GC separations and increased signals in the MS via the SPME analysis of the collected hydrocarbons (Table 1). Proton transfer reaction mass spectroscopic analyses showed a marked increase in the ratio of ethanol (mass 47) to the total mass of all other ionizable VOCs going from $c a$. 0.6 in the wild type strain (C1-4) to ca. 0.8 in C1-4 AZA (grown in the presence of AZA). Strain C1-4B was created by exposure of the fungus to $100 \mu \mathrm{m}$ SAHA and upon removal of 
Table 1 Comparison of VOC production of Hypoxylon sp. Cl-4 (wild type) and its epigenetic variants C1-4 SAHA, C1-4B and C14 AZA*a

Wil

\section{Retention Time}

$5 \quad(\min )$

10

5.22

5.24

6.15

\subsection{6}

6.70

7.25

7.70

7.96

8.03

$15 \quad 8.55$

8.72

8.83

\subsection{5}

$20 \quad 9.62$

9.82

9.93

$$
9.99
$$

\subsection{7}

11.85

$25 \quad 12.17$

12.26

\subsection{0}

12.41

12.55

$\begin{array}{ll}30 & 12.99\end{array}$

\section{1}

13.18

14.22

13.39

$35 \quad 16.45$

16.69

17.14

19.26

19.87

19.87

40

\subsection{3}

20.04

20.18

20.62

21.05

$45 \quad 21.60$

21.89

22.02

23.78

23.83

$50 \quad 24.02$

24.11

24.47

24.77

Compound

Acetaldehyde*

2,4,5-Trimethyl-1,3-dioxolane

Decane*

Undecane, 5-methyl*

$\infty$-Thujene

2-Butenal*

Nonane, 5-butyl-

3,6-Dimethyldecane

1,3,5-Trioxane, 2,4,6-trimethyl-

Dodecane*

Sabinene*

1-Butanol, 3-methyl-, acetate*

1,3-Cyclopentadiene, 5-(1-

methylpropylidene)-

Cyclohexene, 1 (1-propynyl)-

1,3,5-Cyclooctratriene

$\beta$-Myrcene

2-Butenoic acid, ethyl ester, $(E)+-+-$

$\delta$-4-Carene*

4-Octanone*

1,8-Cineole*

1,3,6-Octratriene, 3,7-dimethyl-(transocimene)

2-Butenic acid, 2-methyl-, ethyl ester

Benzene, 1-ethenyl-2-methyl

$\gamma$-Terpinene*

1,3,7-Octratriene, 3,7-dimethyl-

Benzene, 1,2,3,4-tetramethyl

Benzene, 1-methyl-4-(1-methylethyl)-

Benzene, 1-methyl-2-(1-methylethyl)-*

Cyclohexane, 1,2,4-tris (methylene)

$\infty$-Terpinolene*

2-Propanoic acid, butyl ester

Benzene, 2-propenyl

1 - $\beta$-Pinene

1-Phellandrene

2,4-Nonadiene, $(E, E)$ -

Butane, 1,1,3,4-tetrachloro-1,2,2,3,4,4-

hexafluoro-

Linalool

Formic acid

2 - $\beta$-Pinene

Cis-p-2-menthen-1-ol

$\infty$-Guaiene

3-Cyclohexen-1-ol, 4-methyl-1-(1-

methylethyl)-

2-(2-Propenyl)-furan

4-(Cyclopentylidene)-2-butanone

1- $\infty$-Terpineol

Linalyl propionate

$\beta$-Selinene

$\delta$-Guaiene

Camphene

Cyclopentane, 1-methylene-3-(1-

methylethylidene)

Unknowns
Wild type

(C1-4) C1-4 SAHA C1-4B C1-4 AZA Quality match Relative area

MW (\%)

(\%)

Relative area $(\%)$

(\%)

$(\%)$

5

$\begin{array}{rr}44 & 2.40 \\ 116 & 0.7 \\ 142 & \end{array}$

142

$\begin{array}{ll}170 & 0.21\end{array}$

136

$\begin{array}{ll}70 & 0.47\end{array}$

$\begin{array}{ll}184 & 0.19\end{array}$

$\begin{array}{ll}170 & 0.20\end{array}$

$132 \quad 0.58$

170

136

$\begin{array}{ll}130 & 0.40\end{array}$

$\begin{array}{ll}120 & 0.21\end{array}$

0.31

$\begin{array}{ll}1.31 & 2.01\end{array}$

$\begin{array}{ll}2.01 & 0.93\end{array}$

1.22

9.61

1.37

13.18

0.92

2.63

2.63
1.40

0.23

0.23

136
136

\section{4}

128

154

154
136

128

128
118

136
136

136
134

\section{4}

134

120

\section{6}

128

118

136

136

124
302

302

154

46
136
154

136
154
204

154

\subsection{7}

0.55

33.39

0.59

36.77

0.19

$$
0.53
$$

0.31

0.31

3.43

1.86

4.75

0.97

1.50

25.03

0.75

3.39

0.82

32.61

6.73

\subsection{6}

1.19

2.67

4.08

20.81
2.43
1.11
1.60
0.47

0.88

0.75

0.46

108

138

$$
154
$$

$$
210
$$

204

204

136

122

14.54

7.95

1.57

2.12

0.46

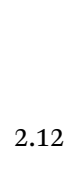

\subsection{3}

1.84

16.39

10.35

0.52

0.24

2.38

0.48

0.90

1.57

${ }^{a}$ The VOCs of these fungi were collected and

a $7 \mathrm{~L}$ flask (10 L flask) for 2 weeks at $22{ }^{\circ} \mathrm{C}$.

90,72

72

87

64

94,94

86

64, 64

90, 90

93, 97, 93, 96

95, 95

25

93

76,94

97, 96, 96

95

76

94

95, 95

$95,95,95,97$

98, 98

$64,64,64$

95

91

72

64

0.46

80

0.36

95

64

70

93

0.50

3.18 
the organism from the epigenetic modulator, it did not revert to the wild type organism. Ultimately, epigenetic modulation of an endophytic fungus can result in phenotypic changes as well as modifications in bioactivity and VOC profiles. Results of this study have implications in understanding why there may be a wide range of VOCs found in various isolates of the same fungus in nature as well as the critical importance that epigenetic factors, possibly associated with plants, may play in VOC production by endophytes. ${ }^{37}$

\subsection{Other endophytes making fuel-related products}

There are an increasing number of endophytic microbes that have been isolated and screened for VOC production. A few are described below that illustrate the potential of this group of organisms as producers of hydrocarbons and other valuable organic compounds.

Daldinia sp. (a Nodulisporium sp.) (EC-12) was isolated as an endophyte of Myroxylon balsamum found in the upper Napo region of the Ecuadorian Amazon. ${ }^{38}$ This organism produced VOCs that have both fuel and biological potentials. Under microaerophilic growth environments, the organism produced 1, 4-cyclohexadiene,1-methyl-, 1-4 pentadiene and cyclohexene, 1-methyl-4-(1-methylethenyl)-, along with some alcohols and terpenoids of interest as potential fuels. The organism was scaled up in an aerated large fermentation flask (9L), and the VOCs trapped by Carbotrap technology and analyzed by headspace solid-phase microextraction (SPME) fiber-GC-MS. Under these conditions a series of alkyl alcohols starting with 3methyl-1-butanol, 2-methyl-1-propanol, 1-pentanol, 1-hexanol, 1-heptanol, 1-octanol, 1-nonanol along with phenylethyl alcohol were detected. ${ }^{38}$ The organism also produced secondary alkyl alcohols, esters, ketones, benzene derivatives, a few terpenoids, and some hydrocarbons. It appears that many of the products have fuel potential. Furthermore, the VOCs of Nodulisporium sp. were active against a number of pathogens causing death to both Aspergillus fumigatus and Rhizoctonia solani and severe growth inhibition produced in Phytophthora cinnamomi and Sclerotinia sclerotiorum within $48 \mathrm{~h}$ of exposure, suggesting a biological role for the VOCs in nature. In addition, the Carbotrapped VOCs somewhat mimicked the bioactivities of the culture itself when certain test organisms were exposed to these VOCs. ${ }^{38}$

An endophytic Nodulisporium sp. (Hypoxylon sp.) has been obtained from Thelypteris angustifolia (Broadleaf Maiden Fern) in a rainforest region of Central America. ${ }^{39}$ The endophyte produced VOCs that have both fuel and biological potential. ; 3-hexanone, 2,4-dimethyl; 2-hexanone, 4-methyl an , 2-one and these account for about $25 \%$ of the VOCs. The most abundant identified VOC was 1,8-cineole, which is commonly detected in this group of organisms. Other
Nodulisporium sp. were selectively active against a number of plant pathogens, and upon a $24 \mathrm{~h}$ exposure caused death to Phytophthora palmivora, Rhizoctonia solani and Sclerotinia sclerotiorum and $100 \%$ inhibition to Phytophthora cinnamomi with only slight to no inhibition of the other pathogens that were tested. ${ }^{39}$ From this work it is becoming increasingly apparent that each isolate of this endophytic organism Nodulisporium spp., including the Daldina spp., Hypoxylon spp. and Annulohypoxylon spp. teleomorphs, seems to produce its own unique set of VOCs with 1,8-cineole as a product common to all.

An unusual Phomopsis sp. was isolated and identified as an endophyte of Odontoglossum sp. (Orchidaceae), growing in rain forests of South America. ${ }^{40}$ This fungus produces a mixture of volatile organic compounds (VOCs) with sabinene (monoterpene), which turns out to be previously known only as a plant product, was identified by the gas chromatography/mass spectrometry under cultural conditions. Other than sabinene, the most abundant compounds recorded in Phomopsis culture were 1-butanol, 3-methyl-, benzeneethanol, 1-propanol, 2-methyl-, benzene methyl, $( \pm)$-, and gymnomitrene. The mixture of volatiles produced by Phomopsis sp. possesses antifungal properties. An artificial mixture of volatiles mimicked the antibiotic effects of the Phomopsis sp., which showed bioactivity against a wide range of test fungi, including Pythium, Phytophthora, Sclerotinia, Rhizoctonia, Fusarium, Botrytis, Verticillium, Colletotrichum, and others. ${ }^{40}$

Larrea tridentata (creosote bush) is a pungent aromatic plant (creosote bush) that grows in the desert regions of Southwestern USA. This plant was studied for its associated endophytes since it produces a plethora of volatile hydrocarbons that are flammable with the rationale that its associated endophytes may also produce identical or related substances. Most commonly used attempts to isolate endophytes from this plant fail. However, in the early spring an endophytic fungus was isolated from the roots, stems and flowers of this plant and characterized as Phoma sp. ${ }^{41}$ This fungus produced a unique mixture of VOCs, including a series of sesquiterpenoids, some alcohols, and several reduced naphthalene derivatives. Trans-caryophyllene, a product in the fungal VOCs, was also noted in the VOCs of this desert plant. The gases of Phoma sp. possess antifungal properties and its bioactivity has a marked resemblance to the bioactivity to that of a methanolic extract of the host plant. Some of the test organisms with the greatest sensitivity to the Phoma sp. VOCs were Verticillium, Ceratocystis, Cercospora and Sclerotinia. ${ }^{41}$ It would appear that this is only one case out of many that may exist in nature, in which a microbial endophyte may mimic the biochemistry of its host in order to survive the conditions of a stressful environment. While both the host and the endophyte do produce at least one hydrocarbon in common, namely trans-caryophyllene, the most abundant fungal product is cis-caryophyllene or humulene (Table 1). While the products of both the host and the endophyte are antifungal it remains to be discovered what the role of each of these sets of products might play in the defense of the host in its native state and what role they play in the ability of the host and its endophyte/pathogen to survive a relative harsh environment. The authors point out that the myriad of VOCs, prominent VOCs produced by this endophyte included 2methyl-1-butanol and phenylethyl alcohol. Also, of interest was the presence of propylcyclohexane, which is a common ingredient of diesel fuel. Furthermore, the VOCs of this isolate of 
such as the alcohols, and other reduced products of this organism have potential as bio-fuels. ${ }^{\mathbf{4 1}}$

\section{5}

\section{Tailoring next-generation biofuels and testing in engine combustion}

While the discovery of promising hydrocarbon producing endophytes is the first step in the process of realizing any domestic utility of these endophytic microorganisms in biofuel production, other important steps need to be made towards scale up, testing, and eventual commercialization of the products. A recent Sandia/US DoE report nicely outlines this process. ${ }^{42}$ The organisms with which the authors of this report worked include the Hypoxylon sp. (Nodulisporium sp.) CI-4A and the Daldinia sp. (also a Nodulisporium sp. EC-12) that are the same organisms mentioned above, along with two other endophytes including Co-27 and EC-38 (also Hypoxylon spp.), both of which were originally isolated in this author's lab. The Sandia report provides a complete outline on how a project might proceed from the field to fungal products being tested in modern engines such as the HCCI (Homogeneous Charge Compression Ignition) (Fig.2). Thus, after isolation and characterization of the endophyte, along with a demonstration of hydrocarbon production, individual compounds may be identified for both scale up and eventual combustion studies (Fig.2). According to the Sandia report, compounds are chosen based on two criteria: prominence in the product stream and lack of existing combustion chemistry knowledge. ${ }^{42}$ In the case of the endophytic fungi mentioned in this review, ketones and cyclic ethers were identified as fitting those criteria and were subjected to combustion chemistry studies. ${ }^{42}$ Fundamental chemical characteristics of representative compounds and detailed

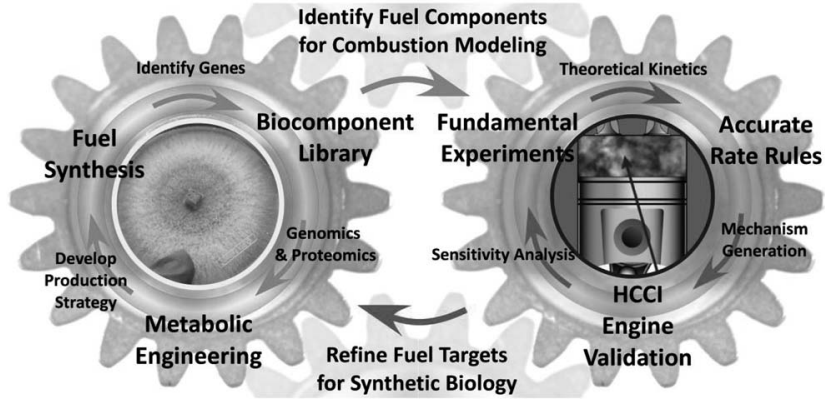

Fig. 2 This simple diagram depicts the framework for collaborative biofuel design as developed in the Sandia project and the illustration is reproduced from their report. ${ }^{42}$ Basically, discovery and synthetic biologists work in close collaboration with combustion researchers to develop fundamental mechanisms for the selection, production and combustion of potential biofuels. Ignition and engine trials then provide feasibility tests for fuels and mixtures and yield recommendations for the bioengineering scale-up of specific metabolic pathways. This coupling of fundamental and applied combustion chemistry and synthetic biology is a strategy to identify and investigate the most promising fuel compounds through mutual feedback. Moreover, the development of combustion models will provide the predictive capability needed for eventual efficient utilization of a new biofuel stream. ${ }^{42}$ theoretical kinetics efforts were combined to develop combustion chemistry models that are predictive of the performance of specific molecules as fuels. ${ }^{42}$ These models are "validated" against HCCI engine measurements, and conclusions about combustion performance were factored into the direction of biofuel production research.

Two possibilities exist for scaling up the desired product(s). The first includes the basic and standard fermentation methodology wherein the selected microbe is subjected to mutationselection for strain improvement and eventual fermentation. Ultimately, carbon balance studies should be conducted to provide information on the percent conversion of substrate to the desired product(s). As an example, using the Hypoxylon sp. strain CI-4A, the \% conversion of a sugar beet waste substrate to hydrocarbons was between $1-2 \%$ dry wt/wt product over a 10 day incubation period [Strobel, Knighton, unpublished results]. Considering the lost cost of the substrate and the high value of the products formed, including ethanol, the process at the present time is economically viable. The second includes the methodology suggested in the Sandia report wherein the endophyte is subjected to complete genome sequencing, and then annotation, followed by targeted metabolic pathway genetic manipulation efforts to increase the chosen product. As a start to this process, each of the Nodulisporium sp. (with both Daldinia sp. and Hypoxylon spp. perfect stages) mentioned in this report was completely sequenced and annotated (Table 2). ${ }^{\mathbf{4 2}}$ Although each of the organisms has a Nodulisporium sp. as its imperfect stage, the size of these fungal genomes ranged from 37.5 Mb to 47.3 Mb (Table 2). Hundreds of genes were identified as those responsible for the breakdown of complex carbohydrates as well as at least 350 genes involved in polyketide and secondary biosynthetic pathways were discovered. Interestingly, the expression of these genes is directly related to the substrates on which the individual fungus is grown. ${ }^{\mathbf{4 2}}$

In the Sandia report the most promising mycodiesel products made by the endophytic fungi (Table 2) under the study were subjected to combustion chemistry analysis. ${ }^{42}$ Ketones and cyclic ethers were identified in the VOC product spectrum from these microorganisms grown on a range of substrates. Fundamental chemistry studies and engine measurements on ketones uncovered some unusual and beneficial characteristics in their ignition chemistry. The cyclic ketones, exemplified by cyclopentanone, are resistant to autoignition and show low reactivity in HCCI engines. The fundamental chemistry experiments show that the initial steps of low-temperature cyclopentanone oxidation are dominated by chain-terminating formation of $\mathrm{HO} 2$. On the other hand, low-temperature oxidation of openchain ketones such as di-isopropyl ketone (DIPK) displayed significant chain-propagating formation of $\mathrm{OH}$ radicals. Furthermore, DIPK showed low-temperature heat release in the HCCI operation above 1.8 bar intake pressure, and, unusually, retained significant sensitivity to temperature even under highly boosted conditions. Consequently, DIPK is a promising fuel for high-load HCCI engines and may be a useful as a knockresistant spark-73 ignition (SI) fuel.

Cineole, a saturated bicyclic ether, is another potential mycodiesel compound and is commonly found among the 
Table 2 Summary of genome sequencing and analysis for various Noduliosporium spp. isolates of the perfect stages of Hypoxylon and Daldinia spp. mentioned in the Sandia report ${ }^{42}$

5 Genomic

Daldinia

$\begin{array}{ll}\text { Daldinia } & \text { Hypoxylon } \\ \text { sp. } & \text { sp. }\end{array}$

EC-12 CI-4A

Hypoxylon
sp.
$\mathrm{Co}^{-27^{a}}$

Hypoxylon

Data sets

Genome size $(\mathrm{Mb})$

Contigs

Number of genes

Gene length (nt)

Transcript length (nt)

Protein length (aa)

Exon length (nt)

Intron length (nt)

Exon frequency (no./gene)

Gene density (no./Mbp)

37.5
641
11173
1811
1638
467
568
94
2.89
298

298

37.7
1044
11712
1761
1600
459
564
89
2.84
311

311

${ }^{a}$ The plant host of Co-27 is Rhizophora sp. and that of EC-38 is Neea floribunda.

fungal metabolism products in Nodulisporium spp. isolates. ${ }^{16}$ Initial studies on the ignition chemistry of cineole shows significant formation of $\mathrm{OH}$ indicating the possibility of lowtemperature heat release in compression-ignition engines. Direct testing in internal combustion engines has shown that it can serve as a fuel additive as mentioned above. ${ }^{32,33}$

\section{The Paleobiosphere}

As a whole, endophytes occupy a unique position among all other microbes in the environment. Their location in the plant, their suppressed growth while present in plant tissues, and their potential to grow and break down plant material after the host has died make them unique. In addition, some endophytes having the capability to make hydrocarbons leads to the prospect that they may have played a role in the origin of crude oil. Thus, the hypothesis that some of these endophytic organisms may have been, in part, responsible for the production of ancient shale-based hydrocarbons (crude oil) has been proposed since it is composed of some of the same classes of hydrocarbons as made by these contemporary endophytic fungi. .11, $14,16,18,37^{-1}$

Oil rich shale beds commonly have large to small bits of petrified plants materials associated with them. ${ }^{14}$ Recently, evidence has emerged for the presence of wood and leaf associated petrified fungi in oil-rich Montana shales and these are located adjacent to some of the most productive wells in the state (Fig. 3). Collectively, these data provide the basis for a reasonable and testable hypothesis that fungi may have contributed to the formation of some of the shale-related VOCs (hydrocarbons) by virtue of their ability to degrade plant materials into hydrocarbons that were ultimately trapped and retained by the unique physical framework of shales and related materials. This is in direct contrast to the hypothesis that crude oil originated via heat and pressure of the earth. ${ }^{43}$

The rationale for this hypothesis is based on the premise that a properly planned and executed experimental test system can be devised in which plant material is degraded by an appropriate fungal endophyte and the VOCs arising therefrom are effectively trapped in a pre-heated processed shale. The design of the system is based on the presumed conditions that mainly existed during the Cretaceous period when the earth very much resembled the present day areas of SE United States from Florida to the Mississippi delta region when the deposition of hydrocarbons seems to have occurred. ${ }^{44}$ As an example, the area, of what is now Eastern Montana and Western North Dakota, when not under-water, was part of a hot humid coastline of rivers, and river deltas bearing dense vegetation that bordered the ancient shallow inland sea. This sea covered an area from the present day Gulf of Mexico to Hudson's bay and included massive shale deposits that underlay and were otherwise associated with the sea. ${ }^{45}$

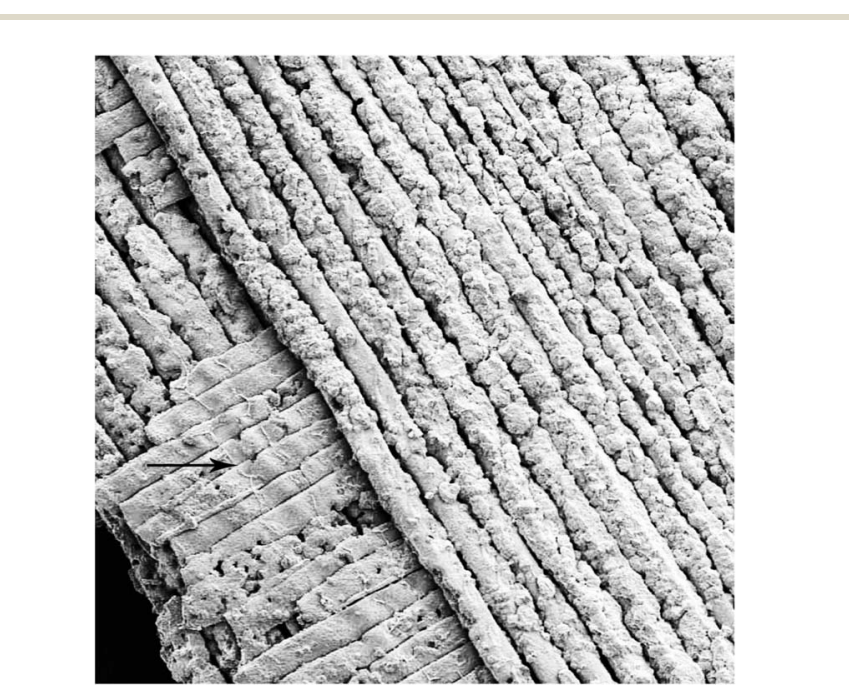

Fig. 3 An SEM of petrified wood gathered at Melstone Mt. near a productive oil well located in bear paw shale. The arrow points to fungal hyphae as they were growing the in the intercellular spaces of the plant. Close examination reveals that $t$ he hyphae are distributed throughout the sample. The hyphae are about 2 microns in diameter (arrow). The specimen dates from the late Cretaceous, 65-70 million years ago. 
In order to test the hypothesis that fungi associated with plants may have produced hydrocarbons that were trapped by what are now ancient shale deposits, a device dubbed a "Paleobiosphere" has been designed. It includes a chamber with the appropriate materials to produce hydrocarbon rich shale by degradation of appropriate extant plant species using an endophytic fungus that produces hydrocarbons. Its design also permits water in varying amounts, filtered sterile air and a Carbotrap to collect all hydrocarbons being produced by the system while not being collected by the "trap" shale. Such an instrument has been built and subjected to testing with all of the necessary ingredients (Fig.4).

The fungus selected for placement in the Paleobiosphere was Annulohypoxylon sp. isolated as an endophyte of Citrus aurantifolia, in the swamps of Florida, which produces, in solid and liquid potato dextrose media, a series of hydrocarbon-like molecules. ${ }^{14}$ Some of these including 1,8-cineole, 2-butanone, 2-methyl-propanoic acid, methyl ester, (1-methylethyl)-benzene, phenylethyl alcohol, benzophenone and azulene,

Fig. 4 The Paleobiosphere used to mimic conditions of the ancient earth. Plant materials, bentonite shale, Annulohypoxylon sp. along with air and water resulted in a mixture of trapped hydrocarbons attached to the trap shale and the Carbotrap on the upper right. ${ }^{14}$ 1,2,3,5,6,7,8,8a-octahydro-1,4-dimethyl-7-(1-methylethenyl), $[1 S-(1 \alpha, 7 \alpha, 8 \mathrm{a} \beta)]-$ and these were the key fungal signature compounds that were followed in an initial Paleobiosphere test. ${ }^{14}$ After 3 weeks of incubation, the volatiles associated with the harvested "Trap Shale" included each of the signature substances as well as other fungal associated products such as some indanes, benzene derivatives, some cyclohexanes, 3octanone, naphthalenes and others. The fungus had produced a series of "Trap Shale" products that were representative of each of the major classes of hydrocarbons in diesel fuel -Mycodiesel. It appears that the initial tests with the Paleobiosphere offered some evidence for a possible origin of hydrocarbons trapped in bentonite shale. Thus, with modifications, it can be imagined that numerous other experiments could also be designed and tested in the Paleobiosphere. Certainly, some of these include the utilization of mixed cultures including some bacterial species and other fungal endophytes along with different plant species and a modification of other physical parameters during the incubation period. The Paleobiosphere experiment certainly does not answer all questions concerning the origins of oil but it offers a glimpse of some of the processes that may have occurred in the formation of this vitally important product. ${ }^{\mathbf{1 4}}$

\section{Concluding remarks}

Presently, it appears that virtually all literature reports of hydrocarbon production by endophytic fungi adequately demonstrate that the phenomenon does occur. The type of hydrocarbon molecules that have been recorded ranges from straight chained/branched hydrocarbons to cycloalkanes to numerous benzene derivatives to many polyaromatic hydrocarbons. They generally represent compounds associated with the four major classes of substances found in diesel fuels. In addition, often in the fungal VOC analyses there are other VOCs that are detected including esters, alcohols, terpenoids, aldehydes and ketones. It is to be remembered that no individual fungus makes all of the compounds found in diesel, but some do make VOCs that are representative of the four diesel classes.

While many of the fungal genomes have been sequenced only one has been annotated and some of the VOCs being produced cannot be accounted for by the genomics of previously known and established gene/enzyme systems. ${ }^{28}$ Thus, it is the case that further biochemical studies on fungi are needed to elaborate the proteomics and genomics of hydrocarbon production. Furthermore, one cannot assume that previously characterized enzymes and genes involved in biosynthetic pathways in plant systems are identical to those of endophytic fungi.

It appears that the next step in commercializing fungi that make hydrocarbons is to increase productivity of these substances. Carbon balance experiments should be done on each candidate fungus. Novel techniques need to be devised that will optimize conversion of substrates to the desired products. This may include the introduction of fermentation procedures that are more favorable to the fungus while taking advantage of the ability of the organism to degrade agricultural 
waste materials. In addition, consideration needs to be made on better utilizing the mycelial structure once it gets established on its substrate. Finally, strain improvement technologies need to be applied to organisms that have been targeted for hydrocarbon production. Finally, if novel fungal VOCs are to be introduced to the fuel market -place they need to be chemically studied and appropriately tested in real engines. It appears as if the time has come for serious consideration and experimental work to be done in order to learn if the approach of using filamentous fungi for fuel and green chemical production has economic merit for the world?

\section{Acknowledgements}

The author acknowledges the support of the National Science Foundation (NSF) Emerging Frontiers in Research and Innovation (NSF-EFRI), Grant No. 0937613 to Dr Brent Peyton and Gary Strobel of MSU and a DoE grant to GAS to carry out much of the work referenced in this paper.

\section{References}

1 G. A. Strobel, Curr. Opin. Microbiol., 2006, 9, 240-244.

2 G. A. Strobel, Phytochem. Rev., 2011, 10, 165.

3 G. A. Strobel, Microbiology Today, 2012, 39, 108-111.

4 C. W. Bacon and J. F. White, Microbial Endophytes, Marcel Dekker Inc., N.Y., 2000.

5 G. A. Strobel and B. Daisy, Microbiol. Mol. Biol. Rev., 2003, 67, 491-502.

6 S. A. Smith, D. C. Tank, et al., PloS 1 Biology, 2008, 3, e3052.

7 V. C. Verma, R. N. Kharwar and G. A. Strobel, Nat. Prod. Commun., 2009, 4, 1511-1532.

8 R. X. Tan and W. X. Zau, J. Nat. Prod., 2001, 18, 448-459.

9 J. Worapong, G. A. Strobel, E. J. Ford, J. Y. Li, G. Baird and W. M. Hess, Mycotaxon, 2001, 79, 67-79.

10 D. Ezra, W. M. Hess and G. A. Strobel, Microbiology, 2004, 150, 4023-4031.

11 G. A. Strobel, B. Knighton, K. Kluck, Y. Ren, T. Livinghouse, M. Griffen, D. Spakowicz and J. Sears, Microbiology, 2008, 154, 3319-3328.

12 F. Zhang, S. Rodriguez and J. Keasling, Curr. Opin. Biotechnol., 2011, 22, 775-783.

13 A. Ahamed and B. K. Ahring, Bioresour. Technol., 2011, 102, 9718-9722.

14 G. A. Strobel, E. Booth, G. Schaible, M. T. Mends, J. Sears and B. Geary, Biotechnol. Lett., 2013, 35, 539-552.

15 M. Stinson, D. Ezra, W. M. Hess, J. Sears and G. A. Strobel, Plant Sci., 2003, 165, 913-922.

16 A. Tomsheck, G. A. Strobel, E. Booth, B. Geary, D. Spakowicz, B. Knighton, C. Floerchinger and J. Sears, Microb. Ecol., 2010, 60, 903-914.

17 M. T. Mends, E. Yu, G. A. Strobel, S. R. U. Hassan, E. Booth, B. Geary, J. Sears, C. A. Taatjes and M. Hadi, J. Pet. Environ. Biotechnol., 2012, DOI: 10.4172/2157-7463.1000117.

18 E. Booth, G. Strobel, B. Knighton, J. Sears, B. Geary and R. Avci, Biotechnol. Lett., 2011, 33, 1963-1972.
19 D. Ezra, J. Jasper, T. Rogers, B. Knighton, E. Grimsrud and G. A. Strobel, Plant Sci., 2004, 166, 1471-1477.

20 W. Lindinger, A. Hansel and A. Jordan, Int. J. Mass Spectrom. Ion Processes, 1998, 173, 191-241.

21 N. Mallette, W. B. Knighton, G. A. Strobel, R. P. Carlson and B. M. Peyton, $A M B$ Express, 2012, 2, 23.

22 D. Banerjee, G. A. Strobel, E. Booth, B. Geary, J. Sears, D. Spakowicz and S. Busse, Mycosphere, 2010, 3, 241-247.

23 C. Song, C. Hsu, and I. Mochida, Chemistry of Diesel Fuels, Taylor \& Francis, N.Y., 2000.

24 B. J. McFee and A. Taylor, Nat. Toxins, 1999, 7, 283-303.

25 M. A. Griffin, D. J. Spakowicz, T. A. Gianoulis and S. A. Strobel, Microbiology, 2010, 156, 3814-3829.

26 N. Mallette, Volatile Fuel and ORganic Compound Production by Ascocoryne sarcoides: Exploration of Environmental Variables and Analytical Methods, PhD thesis, Montana State University, Bozeman, Mt, 2013.

27 G. A. Strobel, B. Knighton, K. Kluck, Y. Ren, T. Livinghouse, M. Griffin, D. Spakowicz and J. Sears, Microbiology, 2010, 156, 3830-3833.

28 T. A. Gianoulis, M. A. Griffen, D. J. Spakaowicz, B. Dunican, C. J. Alpha, A. Sboner, M. Sismour, C. Kodira, M. Egholm, G. Church, M. B. Gerstein and S. Strobel, PLoS Genet., 2012, 8, e1002558.

29 G. A. Strobel, A. Tomsheck, B. Geary, D. Spakowicz, S. Strobel, S. Mattner and R. Mann, Mycology, 2010, 1, 187194.

30 R. T. Hanlin, Illustrated Genera of Ascomycetes, Am. Phytopath. Press, St. Paul, Minnesota, 1997.

31 R. Croteau, W. R. Alonso, A. E. Koepp and M. A. Johnson, Arch. Biochem. Biophys., 1994, 309, 184-192.

32 A. Barton and J. Tjandra, Fuel, 1989, 68, 11-17.

33 K. Sugito, and S. Takeda, US Pat., 4297 109, 1981.

34 E. K. Shwab, J. W. Bok, M. Tribus, J. Galehr, S. Graessle and N. P. Keller, Eukaryotic Cell, 2007, 6, 1656-1664.

35 R. B. Williams, J. C. Henrikson, A. R. Hoover, A. E. Lee and

R. H. Cichewicz, Org. Biomol. Chem., 2008, 6, 1895-1897.

36 R. H. Cichewicz, Nat. Prod. Rep., 2010, 27, 11-22.

37 R. Hassan, G. A. Strobel, E. Booth, B. Knighton, C. Floerchinger and J. Sears, Microbiology, 2012, 158, 465473.

38 M. T. Mends, E. Yu, G. A. Strobel, S. R. U. Hassan, E. Booth, B. Geary, J. Sears, C. A. Taatjes and M. J. Hadi, J. Pet. Environ. Biotechnol., 2012, 3, DOI: 10.4172/2157-7463.1000117.

39 S. R. Hassan, G. A. Strobel, B. Geary and J. Sears, J. Microbiol. Biotechnol., 2013, 23, 29-35.

40 S. K. Singh, G. A. Strobel, B. Knighton, B. Geary, J. Sears and D. Ezra, Microb. Ecol., 2011, 61, 729-739.

41 G. A. Strobel, S. K. Singh, R. U. L. Hassan, A. Mitchell, B. Geary and J. Sears, FEMS Microbiol. Lett., 2011, 320, 87-94. 42 A. M. Gladden, C. A. Taatjes, C. Gao, G. O’Bryan, A. J. Powell, A. M. Scheer, K. Turner, W. Wu and E. T. Yu, Tailoring NextGeneration Biofuels and Their Combustion in NextGeneration Engines, Sandia Report, 2013, 2013-10094.

43 W. L. Russell, Principles of Petroleum Geology, McGraw-Hill Co, N.Y., 1960. 
1

44 T. J. Heck, R. D. LeFever and D. W. Fischer, Overview of the petroleum geology of the North Dakota Williston Basin,

13 North Dakota Geological Survey, 2012.

45 T. M. Finn, M. A. Kirschbaum, S. B. Roberts, S. M. Condon, L. Roberts, and R. C. Johnson, Cretaceous-Tertiary Composite Total Petroleum System (503402), Bighorn
Basin, Wyoming and Montana - Chapter 3 of Petroleum Systems and Geologic Assessment of Oil and Gas in the Bighorn Basin Province, Wyoming and Montana By U.S. Geological Survey Bighorn Basin Province Assessment Team U.S. Geological Survey Digital Data Series DDS-69-V 2010, 2010. 